\title{
Giant myxoid liposarcoma of descending mesocolon origin
}

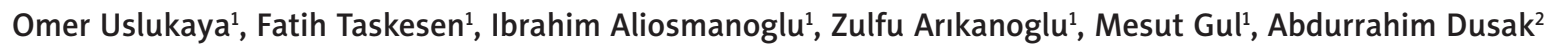 \\ ${ }^{1}$ Department of Surgery, Dicle University Hospital, Faculty of Medicine, Diyarbakir, Turkey \\ ${ }^{2}$ Department of Radiology, Dicle University Hospital, Faculty of Medicine, Diyarbakir, Turkey
}

Prz Gastroenterol 2014; 9 (6): 361-364

DOI: $10.5114 / p g .2014 .47899$

Key words: liposarcoma, mesocolon, sarcoma.

Address for correspondence: Omer Uslukaya Assist. Prof., Department of General Surgery, Dicle University School of Medicine, 21280, Yenisehir, Diyarbakir, Turkey, phone: +90 4122488001 (4754), fax: +90 412248 8523, e-mail: druslukaya@gmail.com

\begin{abstract}
Liposarcomas are one of the most common primary lesions of the retroperitoneal region. They rarely exhibit intra-abdominal location. Because the symptoms emerge later on, they often remain unnoticed until they grow large. Our aim in this paper is to present a case of myxoid liposarcoma of descending mesocolon origin, $40 \mathrm{~cm}$ wide and weighing $7000 \mathrm{~g}$, seen in a 47-year-old male patient. No cases at this weight and radius, originating from descending mesocolon, belonging to the myxoid sub-type, were found in our literature scan.
\end{abstract}

\section{Introduction}

Soft tissue sarcomas make up less than $1 \%$ of all malignancies. The most common type is liposarcoma [1]. Liposarcomas are usually located in the lower extremities (56\%) and the retroperitoneal region (20\%). They are rarely located abdominally [1]. Symptoms of abdominal and retroperitoneal tumours usually emerge late and remain unnoticed until they grow large. There are five histological sub-types, the most common one being myxoid [2]. The presented case is of a myxoid liposarcoma, $40 \mathrm{~cm}$ wide, weighing $7000 \mathrm{~g}$, originating from the descending mesocolon of a 47-year-old male patient. No cases at this weight and radius, originating from descending mesocolon, belonging to the myxoid sub-type, were found in our literature scan.

\section{Case report}

A 47-year-old male patient presented at our policlinic with a complaint of stomachache, lasting for 3 months, and swelling of his abdomen. After physical examination and the use of imaging techniques, he was admitted to our clinic.

In the physical examination, there was a painless, immobile mass that almost filled the abdomen with definite borders with palpation, with intermediate hardness.
A 64-slice dynamic contrast enhanced computerised tomography (CT) scan demonstrated a $36 \mathrm{~cm} \times 27 \mathrm{~cm}$ $\times 37 \mathrm{~cm}$ giant mass, arising in the mesentery and extending to the abdomen and pelvis, with well circumscribed planes, containing a heterogeneous hypodensity with slightly irregular enhancement that filled the entire abdominal and pelvic cavity with no metastases, and which caused anterior compression of both kidneys and compression of the inferior vena cava and renal veins and distorted the bowels. The bowel loops were distributed across the tumour between the tumour and the abdominal wall (straddling sign), due to the mesenteric location of the tumour. Finally, the CT scan also showed the presence of external impression vena cava inferior and renal veins. Based on the radiological data, the clinical diagnosis was made as mesenteric giant liposarcoma. There was a minimal amount of ascites in the Douglas' space (Figure 1). Laboratory values and tumour markers were within normal boundaries.

The patient was operated under elective circumstances. In exploration, it was seen that the mass originated from the descending mesocolon and exhibited no retroperitoneal extensions pushed all intra-abdominal organs to the left of the middle line. The mass was completely excised, and because it originated from descending mesocolon, segmental resection and anastomosis were applied. The excised mass was approximately 

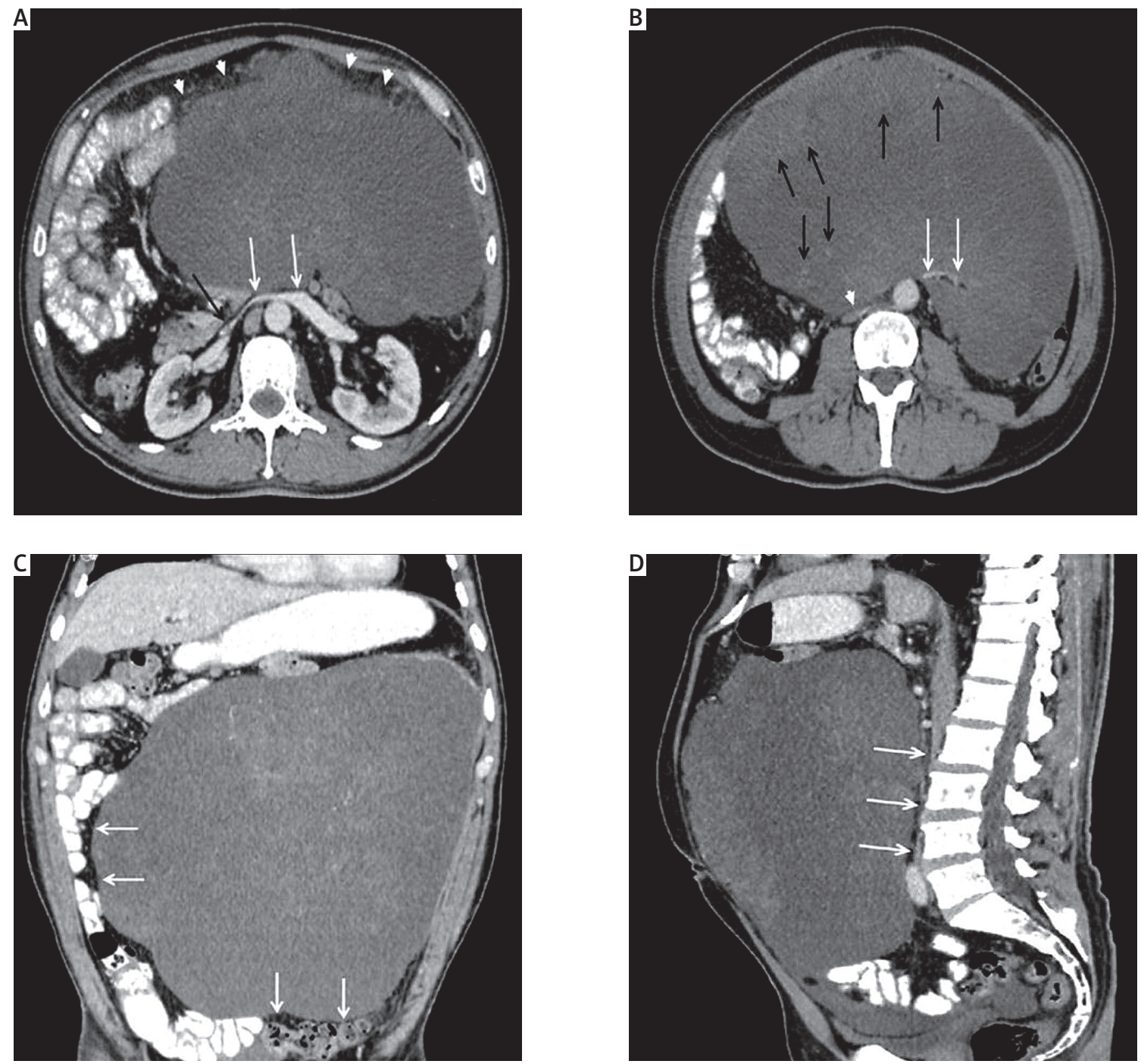

Figure 1. Contrast enhanced axial (A, B), coronal (C), and sagittal (D) computerised tomography scan demonstrates the presence of a giant mass distending the abdomen and pelvis. Heterogeneously enhancing (black arrows in B) lesion placed anterior to the mesenteric vessels (arrows). Coronal image demonstrates the bowel loop (straddling sign) (arrows in C) and mesenteric fat (arrowhead in A) over the mass. Anterior compression of the renal veins and inferior vena cava (arrows in A, arrowheads in B, and arrows in D)

$40 \mathrm{~cm}$ wide, weighed $7000 \mathrm{~g}$, was greyish-brown, and had a fairly smooth surface and a lobule appearance (Figures 2 A, B).

In histopathological examination, small "spindle-like/ovoid" and sometimes star-like shaped small atypical cells, which were stained positive with vimentin and S-100 in immunohistochemical staining (Figure $3 \mathrm{~A}$ ), showed no immunoreactions with CD117 and Desmin, resembling the histomorphology of angiomyxoma in some focuses. Widespread myxoid changes were observed (Figure 3 B). The tumour cells exhibited an intermediate level of atypicality, and Ki-67 proliferation index was evaluated as 3-4\% (Figure 3 C). As a result of pathologic examination it was interpreted as "lowgrade myxoid liposarcoma".

The patient, who had no problems, was discharged on the eighth day after operation. In the follow-ups that were continued for the following year, no clinical or radiological pathologies were seen.

\section{Discussion}

Liposarcomas make up $20 \%$ of soft tissue sarcomas [1]. They are usually seen in males, in the fifth and seventh decades of life [1]. They are most commonly locat- 

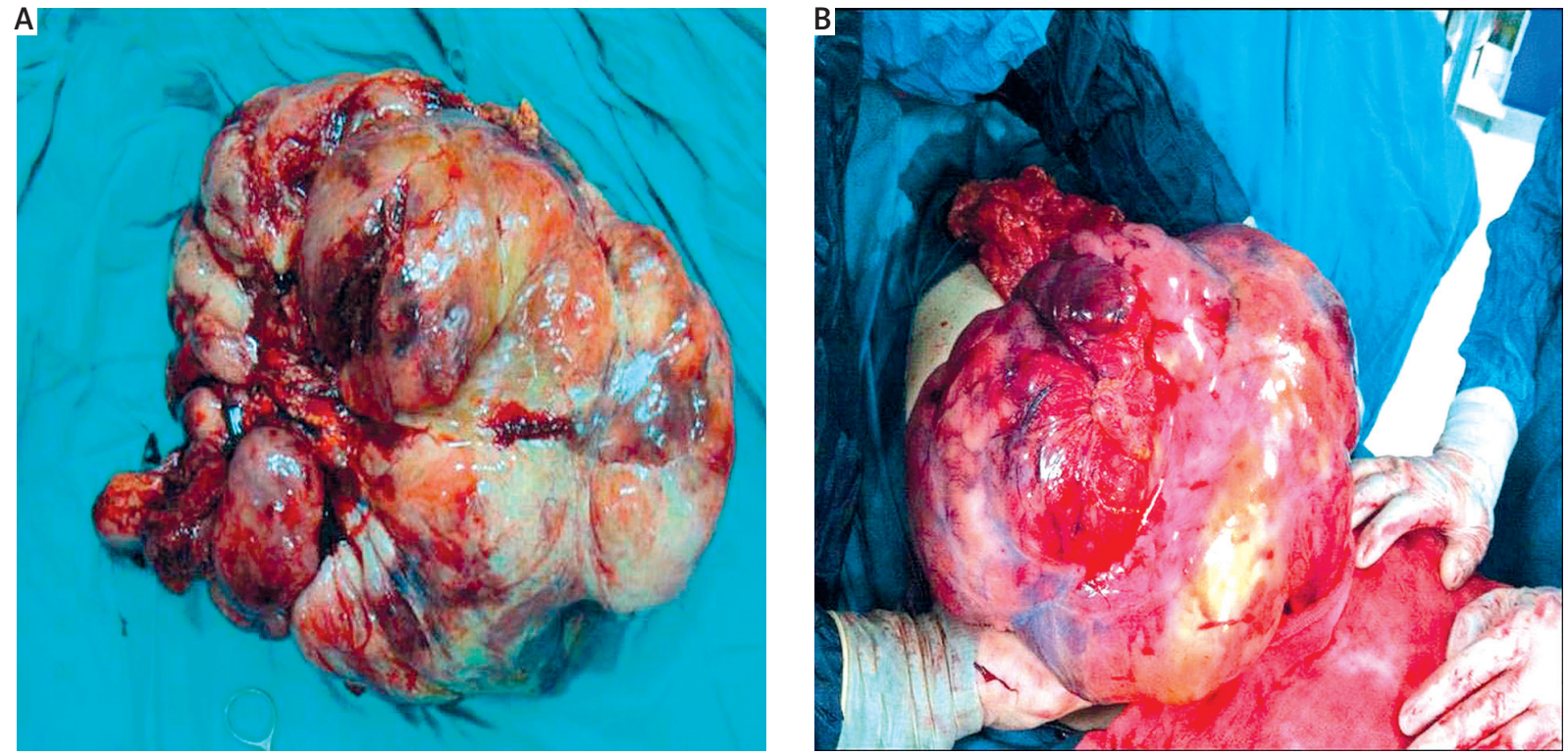

Figure 2. Macroscopic view of liposarcoma
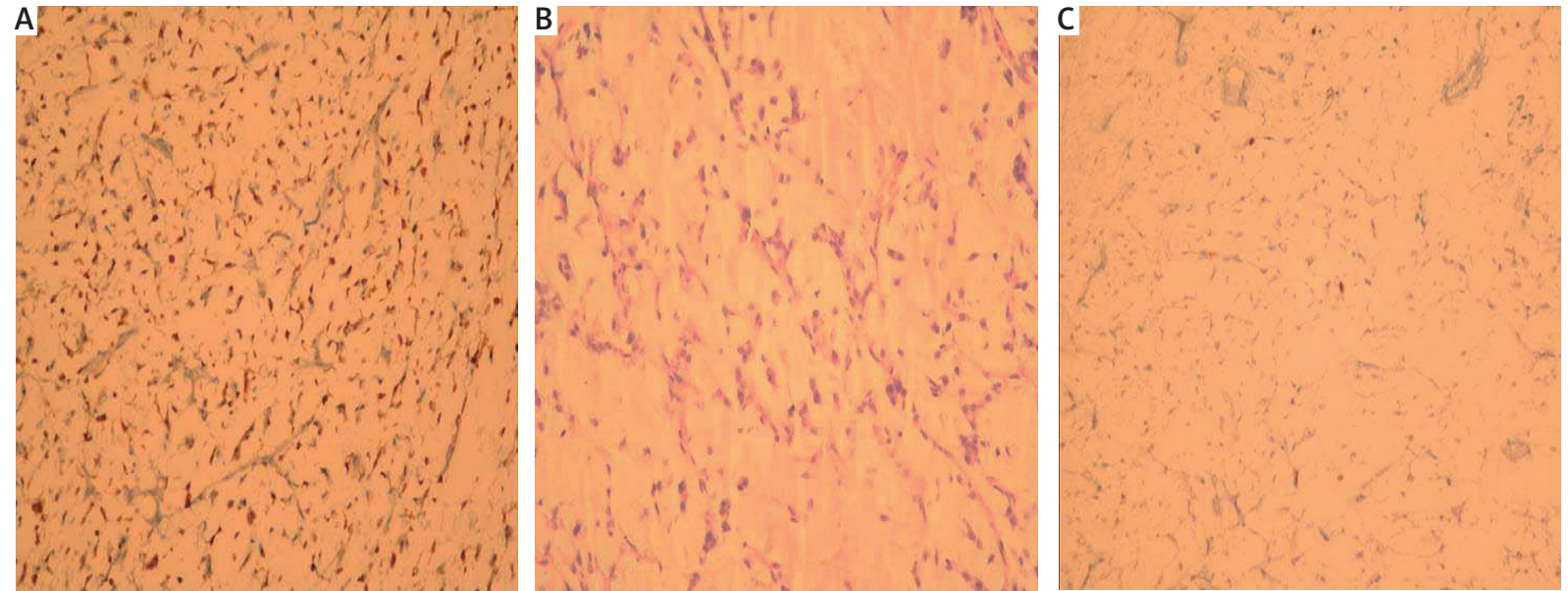

Figure 3. A - Diffuse S-100 positivity in the tumour (immunoperoxidase stain, 200x). B - Spindle-ovoid shaped atypical cells within a myxoid stroma (H + E stain, 200x). C - Ki-67 staining (arrows) in the tumour (immunoperoxidase stain, 200x)

ed in the extremities and the retroperitoneal area [1]. Liposarcomas primarily originating from the mesocolon are quite rare [1].

Patients with abdominally located liposarcomas usually seek advice with gradual increase around the abdomen, weight loss, constipation, stomachache, and a mobile mass in the abdomen [1]. Symptoms of liposarcomas located in retroperitoneal and abdominal zones do not usually emerge until they reach a large size, due to their non-specific symptoms. Similarly, our patient presented with stomachache and a mass in the abdomen. In the literature, large intra-abdominally located masses weighing
8-9 kg have been reported [1, 3]. In our case, the mass weighed $7000 \mathrm{~g}$ and was located intra-abdominally.

Radiological imaging provides useful information for diagnosis. Computed tomography scan can demonstrate the features of liposarcoma, such as tumour inhomogeneity, poor margin, heterogeneous hypodensity, and contrast enhancement that changes with histological grade. Mesenteric lipodystrophy is an important differential diagnosis, which is a self-limiting disease with the feature of spontaneous regression. In the present case, the necrotic component and mass effect pointed towards the radiological differential diagnosis of either 
a gastrointestinal stromal tumour or sarcoma. Computed tomography appearances of differentiated liposarcoma can be to the same as normal fatty tissue. Liposarcomas are moderately vascular, cause displacement of the vessels, and may show irregular, fine tumour vessels and areas of tumour stain. A fat plane around of the tumour suggested that it originated in mesentery [4]. In the present case the bowel loops were displaced laterally and inferiorly, which is described as a straddling sign of bowel loops.

The World Health Organization categorises liposarcomas into five histological types: myxoid, well differentiated, dedifferentiated, pleomorphic, and mixed type [2]. The pathological type seen in this case, myxoid, is the most common one (50\%) [5].

Prognosis depends on parameters such as histological type, differentiation degree, and surgical excision. Generally, while pleomorphic, round cell, and dedifferentiated types exhibit poor prognosis, well differentiated and myxoid types exhibit good prognostic properties [6]. Five-year survival rates in myxoid and well differentiated types are, respectively, $77 \%$ and $85 \%$, and in round cell and pleomorphic types, respectively, 18\% and $21 \%$ [7]. Myxoid liposarcomas carry higher risk of metastasis [1]. Sato et al. showed that tumour sizes exceeding $20 \mathrm{~cm}$ significantly relate with poor prognosis [8]. In light of these studies, close follow-up was suggested, considering that the prognosis of our patient could be poor due to the wide lesion (approximately $40 \mathrm{~cm})$.

The preferred treatment method for these patients is to apply wide surgical resection, achieving negative surgical margin [1,9]. The real benefits of adjuvant treatments are still debated and long-term survival effects have not been proven yet [1,3]. Adjuvant chemotherapy and radiotherapy are suggested for patients with high relapse risk [3]. In this case, chemotherapy after wide surgical resection was applied.

Liposarcomas should be kept in mind in the differential diagnosis of intra-abdominal masses. Considering liposarcomas before operation will lead to excising the mass more carefully with negative surgical margin.

\section{References}

1. Ciraldo A, Thomas D, Schmidt S. Giant abdominal liposarcoma: a case report. Int J Urol 2000; 1: 1.

2. Fletcher CDM, Unni KK, Mertens F. World Health Organization Classification of tumours. Pathology and genetics of tumors of soft tissue and bone. IARC Press, Lyon 2002; 227-32.

3. Cerullo G, Marrelli D, Rampone B, et al. Giant liposarcoma of the mesentery: report of a case. Ann Ital Chir 2007; 78: 443-5.

4. Jain SK, Mitra A, Kaza RCM, et al. Primary mesenteric liposarcoma: an unusual resentation of a rare condition. J Gastrointest Oncol 2012; 3: 147-50.
5. Inoue K, Higaki Y, Yoshida H. Giant retroperitoneal liposarcoma. Int J Urol 2005; 12: 220-2.

6. Newlands SD, Divi V, Stewart CM. Mixed myxoid/round cell liposarcoma of the scalp. Am J Otolaryngol 2003; 24: 121-7.

7. Tanaka M, Hizawa K, Tonai M. Liposarcoma: a clinicopathological study on 136 cases based on the histologic subtyping of WHO. Jpn J Cancer Clin 1974; 20: 1036-47.

8. Sato T, Nishimura G, Nonomura A, et al. Intra-abdominal and retroperitoneal liposarcomas. Int Surg 1999; 84: 163-7.

9. Mendenhall WM, Zlotecki RA, Hochwald SN, et al. Retroperitoneal soft tissue sarcoma. Cancer 2005; 104: 669-75.

Received: 22.05 .2012

Accepted: 28.12.2012 